\title{
Sparc: a sparsity-based consensus algorithm for long erroneous sequencing reads
}

Chengxi Ye, Sam Ma

Motivation: The third generation sequencing (3GS) technology generates long sequences of thousands of bases. However, its current error rates are estimated in the range of 15$40 \%$, significantly higher than those of the prevalent next generation sequencing (NGS) technologies (less than 1\%). Fundamental bioinformatics tasks such as de novo genome assembly and variant calling require high quality sequences that need to be extracted from these long but erroneous $3 G S$ sequences. Results: We describe a versatile and efficient linear complexity consensus algorithm Sparc to facilitate de novo genome assembly. Sparc builds a sparse k-mer graph using a collection of sequences from a targeted genomic region. The heaviest path which approximates the most likely genome sequence is searched through a sparsity-induced reweighted graph as the consensus sequence. Sparc supports using NGS and 3GS data together, which leads to significant improvements in both cost efficiency and computational efficiency. Experiments with Sparc show that our algorithm can efficiently provide high-quality consensus sequences using both PacBio and Oxford Nanopore sequencing technologies. With only 30x PacBio data, Sparc can reach a consensus with error rate $<0.5 \%$. With the more challenging Oxford Nanopore data, Sparc can also achieve similar error rate when combined with NGS data. Compared with the existing approaches, Sparc[i] calculates the consensus with higher accuracy, uses $80 \%$ less memory and time, approximately. Availability: The source code is available for download at https://github.com/yechengxi/Sparc 


\title{
Sparc: a sparsity-based consensus algorithm for long erroneous sequencing reads
}

\author{
Chengxi $\mathrm{Ye}^{1, *}$, Zhanshan (Sam) Ma ${ }^{2, *}$ \\ ${ }^{1}$ Department of Computer Science, University of Maryland, College Park, MD 20740, USA. \\ ${ }^{2}$ Computational Biology and Medical Ecology Lab, State Key Laboratory of Genetic Resources and \\ Evolution, Kunming Institute of Zoology, Chinese Academy of Sciences, Kunming, Yunnan 650223, \\ China.
}

\begin{abstract}
Motivation: The third generation sequencing (3GS) technology generates long sequences of thousands of bases. However, its current error rates are estimated in the range of $15-40 \%$, significantly higher than those of the prevalent next generation sequencing (NGS) technologies (less than 1\%). Fundamental bioinformatics tasks such as de novo genome assembly and variant calling require high quality sequences that need to be extracted from these long but erroneous $3 G S$ sequences. Results: We describe a versatile and efficient linear complexity consensus algorithm Sparc to facilitate de novo genome assembly. Sparc builds a sparse $k$-mer graph using a collection of sequences from a targeted genomic region. The heaviest path which approximates the most likely genome sequence is searched through a sparsity-induced reweighted graph as the consensus sequence. Sparc supports using NGS and $3 G S$ data together, which leads to significant improvements in both cost efficiency and computational efficiency. Experiments with Sparc show that our algorithm can efficiently provide high-quality consensus sequences using both PacBio and Oxford Nanopore sequencing technologies. With only 30x PacBio data, Sparc can reach a consensus with error rate $<0.5 \%$. With the more challenging Oxford Nanopore data, Sparc can also achieve similar error rate when combined with NGS data. Compared with the existing approaches, Sparc calculates the consensus with higher accuracy, uses $80 \%$ less memory and time, approximately.
\end{abstract}

Availability: The source code is available for download at https://github.com/yechengxi/Sparc

\section{INTRODUCTION}

Three generations of DNA sequencing technologies have been developed in the last three decades, and we are at the crossroads of the second and third generation of the sequencing technologies. Compared with the previous generations, the third generation sequencing (3GS) can provide reads in the range of 5-120 kilo-bases in one fragment. However, at present, the reported error rates are $\sim 15 \%$ with PacBio sequencing (Koren et al. 2012), and as high as $\sim 40 \%$ with Oxford Nanopore

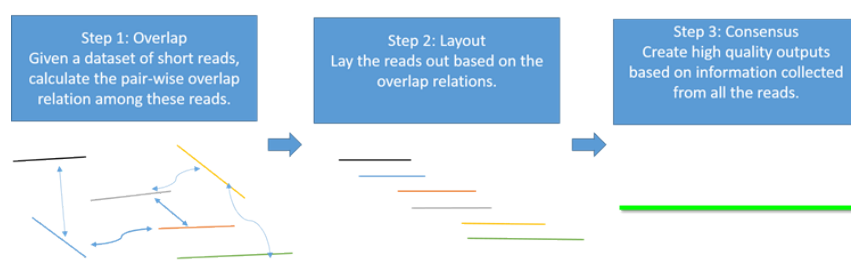

74 Fig. 1. A standard Overlap-Layout-Consensus genome 75 assembly pipeline.

77 The consensus algorithm is critical for genome assembly

78 for multiple reasons. Firstly, the consensus algorithm is a

79 necessary part of an assembler to produce high quality 80 outputs. Secondly, recent assembly advancements resort to 81 an error correction procedure (Au et al. 2012; Hackl et al. 82 2014; Koren et al. 2012; Salmela \& Rivals 2014) to raise the 83 per-base accuracy in the input sequences. The consensus 84 algorithm can also be used to polish each individual read; 85 those corrected reads are usually used as high quality inputs, 86 and are fed into existing Overlap-Layout-Consensus based 87 assemblers that require accurate inputs (Huang et al. 2003; 
88 Mullikin \& Ning 2003; Myers et al. 2000). In the first 89 scenario, each draft assembly contig (or called backbone) is 90 used as the target, and all the reads that align to this 91 backbone are used as the query sequences to raise the 92 quality of the backbone. In the second scenario, each long 93 erroneous read is treated as the target; sequences from either 94 NGS or 3GS may be used as the query sequences. Utilizing 95 NGS data together with 3GS data, known as the hybrid 96 assembly approach, has been a widely adopted assembly 97 strategy since the birth of 3GS technologies. Since the NGS 98 short read data cost significantly less and has higher 99 accuracy, incorporating them can potentially reduce the cost 100 and computational burden of the whole assembly pipeline 101 (Ye et al. 2014). Nevertheless, due to the lack of efficient 102 consensus algorithm, this consensus step is usually 103 circumvented by simpler approaches such as replacing 104 regions in the target sequences with the NGS reads or 105 assemblies. Unfortunately, errors in the NGS sequences may

106 corrupt the originally correct $3 \mathrm{GS}$ sequences and create 107 unwanted consensus errors in the final assembly. Finally, it 108 is also noteworthy that the consensus step in genome 109 assembly pipelines often takes the largest portion of the 110 computational time (Berlin et al. 2014; Chin et al. 2013; Lee 111 et al. 2014). Therefore, an efficient consensus algorithm can 112 significantly accelerate the whole genome assembly process.

113 Traditionally, multiple sequence alignment, known to be a

114 computationally challenging task is used to find the layout

115 and construct a sequence alignment graph (Edgar 2004;

116 Larkin et al. 2007; Lee et al. 2002; Rausch et al. 2009).

117 Alignments are refined and clustered to infer the alignment

118 profile as the consensus in the target region. The higher

119 error rates lead to much higher complexities with these

120 traditional approaches. To lower the complexity, researchers

121 have tried to simplify the multiple sequence alignment by

122 aligning all query sequences to a backbone sequence and

123 creating a multigraph representing the alignment graph

124 (Chin et al. 2013). Each nucleotide base leads to a graph

125 node. Graph simplifications are applied to merge the

126 multiple edges, and the best scored path is found as the

127 consensus sequence. For NGS data, a similar strategy using

128 the de Bruijn graph (DBG) has been developed (Ronen et al.

129 2012) to correct the assembly errors in single cell sequence 130 assembly.

131 In this work, we borrow wisdom from the well-known de 132 Bruijn/k-mer graph (Hannenhalli et al. 1996; Pevzner et al.

133 2001; Ronen et al. 2012) and design a simpler graph

134 formulation of the consensus problem for 3GS data. A

135 general and versatile 'Sparc' algorithm is developed to

136 polish long erroneous draft assembly sequences. Sparc

137 builds a regular directed acyclic graph (i.e., non-multigraph)

138 directly from the sequences. Each node in our regular graph

139 is a $k$-mer. $k$-mers that appear in the same location are

140 merged on the fly when constructing the graph to reduce

141 memory consumption. The graph is also allowed to be 142 'sparse' (Ye et al. 2012) to further avoid using excessive 143 memory caused by false $k$-mers. The links/edges between 144 the $k$-mers are constructed when feeding in each read. Edge 145 weight represents the reliability of the link. Intuitively, a 146 path with the highest sum of edge weights is a good 147 approximation of the consensus. This path is therefore 148 searched and regarded as the algorithm output. Details can 149 be found in the next section. Sparc can provide superb 150 results at low memory, without utilizing any other graph 151 simplification techniques. Due to its simplicity, the
152 algorithm is five times faster and uses five times less 153 memory space compared to a major 3GS consensus program 154 PBdagcon (Chin et al. 2013). Moreover, since the accuracy 155 of the prevalent NGS sequencing data $(>99 \%)$ is 156 significantly higher than that of the NGS data, it is desirable

157 to use the cheaply available NGS to substitute some portion 158 of the costly $3 \mathrm{GS}$ data. In this scenario, a consensus program 159 capable of utilizing different types of data is highly

160 anticipated and should provide higher quality output. Sparc

161 is designed to meet these requirements and it provides high

162 quality results in the hybrid setting for major 3GS

163 technologies.

\section{METHODS}

165 Sparc consists of the following four simple steps: $(i)$ 166 Build an initial position specific $k$-mer graph (Ye et al. 167 2012) using the draft assembly/backbone sequence. (ii)

168 Align sequences to the backbone to modify the existing

169 graph. (iii) Adjust the edge weights with a sparse penalty.

170 (iv) Search for a heaviest path and output the consensus

171 sequence. The pseudo-code can be found in Fig. 2

172

\section{$173 \quad 2.1 \quad$ Building the initial graph}

174 Sparc uses $k$-mers to encode the local structure of the 175 genomic region. It takes a preassembled draft 176 assembly/backbone to build an initial $k$-mer graph (Fig. 3a). 177 The $k$-mers in different positions of the backbone are 178 treated as independent nodes. Therefore this initial graph is 179 a linear list of the $k$-mer nodes. Note that this is a major 180 difference from the popular de Bruijn graphs in genome 181 assembly. Same $k$-mers in different locations are collapsed 182 in a de Bruijn graph. To deal with the long reads, it is 183 important to differentiate $k$-mers by their positions in the 184 genome. In the consensus context, $k$-mers are position 185 specific; different positions are treated independently. It is 186 noteworthy that allocating $k$-mers in each location can take 187 a large amount of memory, especially in the next stage. To 188 circumvent this problem, we allow constructing a sparse $k$ 189 mer graph (Ye et al. 2012) by storing a $k$-mer in every $g$

Sparc Consensus Algorithm:

1. Given the backbone sequence and $k$, $g$, build a position specific $k$-mer graph: sample every $g$ $k$-mers, and record their location in the backbone sequence.

2. Align each query sequence to the existing graph.

2.1 If a query region suggests a novel path/variant then create a branch and allocate new $k$-mer nodes and links between these nodes.

2.2 If a query region perfectly aligns to an existing region in the graph then increase the edges weights in the region.

3. Reduce all the edge weights in the graph by $\max \left(c, t^{\star} \mathrm{Cov}\right)$.

4. Use a Dijkstra-like breadth first search to search for a heaviest path as the final consensus sequence.

bases, which reduces the memory consumption up to $1 / g$.

191 We also record the edge links between the $k$-mer nodes. 
192

193

194

195

196

197

198

199

200

\section{1}

202

\subsection{Aligning sequences to the backbone and building the whole graph}

203 Sequences that align to the backbone sequence provide 204 rich information about the ground truth sequence. Ideally, a 205 most likely genome sequence should be searched as the 206 consensus given all the input sequences. However, utilizing 207 the multi-sequence information comprehensively requires 208 computationally expensive operations such as pair-wise 209 alignment of all the related sequences (Edgar 2004; Larkin 210 et al. 2007; Lee et al. 2002; Rausch et al. 2009). Here we 211 adopt a similar but simpler strategy in PBdagcon (Chin et 212 al. 2013), by aligning all the sequences to the backbone, 213 and modify the existing graph according to the alignments. 214 Rather than creating an intermediate graph that needs to be 215 refined or simplified (Chin et al. 2013; Rausch et al. 2009), 216 we construct the final graph on the fly. We borrow the 217 wisdom from constructing a de Bruijn/k-mer graph 218 (Pevzner et al. 2001; Ye et al. 2012): (i) If a query region 219 suggests a novel path/variant, we create a branch and 220 allocate new $k$-mer nodes and edges between these nodes. 221 An example can be found in the upper half of Fig. 3b, 222 when we align the last six bases of Seq1 to the existing 223 graph. In this example, two new edges ACC and AAA with 224 multiplicity 1 , and one $k$-mer node $C C$ are allocated. (ii) If 225 a query region perfectly aligns to an existing region in the 226 graph, we increase the edges weights in the region without 227 allocating new nodes. Examples can also be found in Fig. $2283 \mathrm{~b}$. When aligning the first five bases of Seq1 to the 229 existing graph, nodes AC, GG and edge TGG are merged 230 implicitly with the ones created by the original backbone, 231 the edge weights are increased by 1 . When aligning the last 232 six bases of Seq2 to the existing graph, the nodes and

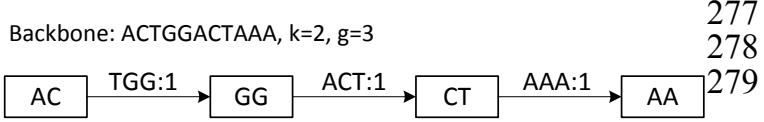

(a)

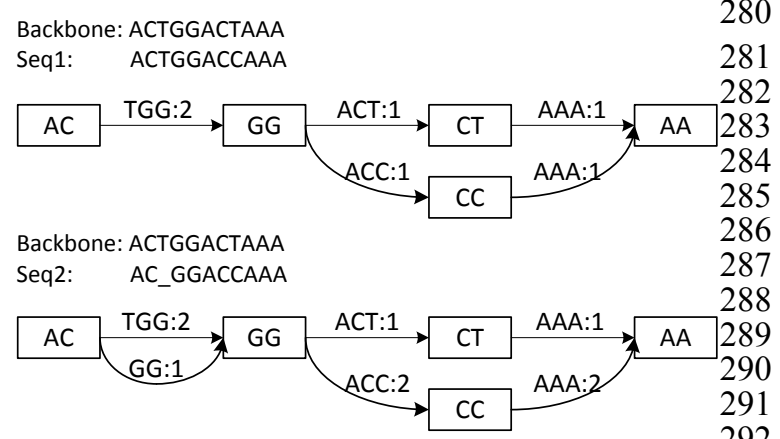

(b)

Find max path to build consensus sequence: ACTGGACCAAA

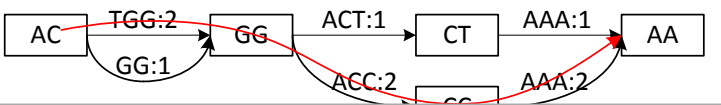

Peer reviewing PDF | (2015:09:6812:3:F:REVIEW 23 Mar 2016) (c)
233 edges are merged implicitly with the ones created by Seq1, 234 the edge weights are changed accordingly. As previously 235 mentioned, this construction process shares similarity with 236 the construction of a de Bruijn graph, but the nodes in our 237 graph are differentiated by their $k$-mers and their positions 238 in the backbone. In addition, Sparc is designed to facilitate 239 hybrid assembly, and can leverage more weight to the high 240 quality data. When different types of sequencing data are 241 available, higher weights can be assigned to the more 242 reliable edges. The resulting $k$-mer graph contains rich 243 information about the underlying genomic region. Next we 244 describe another simple technique to extract the most likely 245 sequence as the consensus output.
246

247

248

249

250

251

252

253

254

255

256

257

258

259

260

261

263

264

265

266

267

268

269

270

271

272

273

274

275

276

277

278

280

281

282

284

286

287

288

290

292
Fig. 3. A toy example of constructing the position specific sparse $k$-mer graph. (a) The initial $k$-mer graph of the backbone. (b) Adding two sequences to the graph. (c) The heaviest path representing the consensus is found by graph traversal (original weights are used in this example).

\subsection{Adjusting the weights of the graph}

Intuitively, a path in the $k$-mer graph is likely to be genuine if it is supported by multiple sequences. Based on this intuition, a path with the highest confidence, i.e. the largest sum of edge weights should be a good approximate of the "ground truth" sequence. However, a direct search for this path may result in an erroneous output. A simple example is a long insertion error: the sum of weights of this erroneous path is higher even though there is only one supporting sequence. To circumvent this type of error, we subtract the edge weight by a small amount. This amount is adaptively determined with consideration of the sequencing coverage in each region. A fixed weight penalty is initially set to be $c=1 \sim 3$; this shares the same heuristic with the parameter setting in existing de Bruijn graph assemblers. These assemblers remove false positive $k$-mers/links by detecting and removing infrequent $k$-mers/links in the dataset. We make this penalty adaptive to the sequencing coverage by introducing an adaptive threshold $\mathrm{t}=0.1 \sim 0.3$ of the average backbone multiplicity. The final penalty is the larger of the two. In all experiments, we fix these two parameters to be $\mathrm{c}=2, \mathrm{t}=0.2$. This penalty technique, also known as soft thresholding (Mallat 2008), is equivalent to put an $l^{1}$-penalty on the edge weights. With this sparse penalty, the low coverage long insertion errors will be less likely to be favored compared to the genuine sequences.

\subsection{Output the heaviest path as the consensus}

The graph constructed in Section 2.2 is a directed acyclic graph, and this allows us to search for a heaviest path with the adjusted weights. To do this, a Dijkstra-like breadthfirst search is adopted to traverse the directed graph from the starting node to the ending node of the backbone. All edges before node $i$ in the backbone must be traversed before we start to traverse the edges starting from node $i$. For each node $i$, the heaviest path to it and its previous node are recorded. After the full traversal, a backtrack from the highest scored node in the backbone reports the highest scored path. This sub-path is output as the consensus path (Fig. 3c). 


\subsection{Implementation Details}

The complexities of the above procedures described in 350 subsections 2.1-2.4 all are linear to the data size. Currently 351 Blasr (Chaisson \& Tesler 2012) is called to provide long 352 read alignment information. Sparc takes a backbone file in 353 fasta format and the Blasr alignment results as inputs. To avoid multiple placement of a query read, each read is mapped to one best matching region in the backbone. Reusing the consensus result as the input and iteratively running the consensus algorithm helps to improve the accuracy even more. Sparc allows for taking different $k$ mer sizes $(k)$ and skip sizes $(g)$. Constructing a sparse graph by skipping every $g k$-mers is beneficial since it consumes $1 / g$ memory and has a similar resolving power with a dense graph using $(k+g / 2)$-mers (Ye et al. 2012). As in the de Bruijn graph, the $k$-mer size defines the anchor size when we analyze the alignments. A large number helps to distinguish between the repeats and avoids merging unrelated sequences. However, since the $3 \mathrm{GS}$ data is usually of high error rates, a smaller $k$-mer size ensures that the weaker alignments still share enough $k$-mers. A tradeoff has to be made here and we find it is necessary to set this number smaller than in most de Bruijn graphs. In our experiments we have found that using $k=1-3, g=1-5$ is sufficient for practical purposes. When high quality NGS data is available, the edges supported by the high quality data shall be treated with higher priority. A parameter $b$ is introduced to selectively increase the weights of the reliable edges by a small amount $(b=5 \sim 10)$ to provide better cues for the correct path.

\section{RESULTS}

Sparc has been tested on a variety of datasets. Here we demonstrate the test results from two PacBio datasets (http://schatzlab.cshl.edu/data/ectools/) and one Oxford Nanopore dataset (http://gigadb.org/dataset/100102). Sparc is designed to be a base-level consensus algorithm. While there are platform-specific ones that take into account signal processing-level information such as Quiver (for PacBio), and Nanopolish (for Oxford Nanopore), these programs usually take the outputs of the base-level ones as inputs to further improve the accuracy. As a fair comparison, we demonstrate results side-by-side with the most similar program to ours, which is PBdagcon (Chin et al. 2013). PBdagcon is the major module that is intensively used in HGAP (Chin et al. 2013) and MHAP (Berlin et al. 2014) pipelines to correct reads and generate consensus using base-level information. We therefore show the comparative results of both programs on these datasets. Both programs are fed with the same input data. We generate assembly backbones and collect the related reads for each backbone using DBG2OLC (Ye et al. 2014). Blasr (Chaisson \& Tesler 2012) is called (with option $-\mathrm{m} \mathrm{5)}$ to obtain the alignments. The final consensus error rates are calculated using the dnadiff function in MUMmer 3 (Kurtz et al. 2004). All experiments are conducted on a workstation with AMD Opteron 2425 HE CPUs (@)
378

379 380
$800 \mathrm{MHz}$ frequency). In some experiments we provide both NGS data and 3GS data. In these 'hybrid' settings 50x Illumina assembly contigs by SparseAssembler (Ye et al. 2012) are included and the edge weights are increased by $\mathrm{b}=5 \sim 10$. 50x Illumina data provides moderate coverage to allow off-the-shelf de Bruijn graph based assemblers to assemble high quality contigs.

On PacBio datasets, we set $k=1, g=1$, and run the consensus algorithms for four rounds. The per-base error rates after 1/2/4 rounds are reported as Err1, Err2 and Err4 in Tables $1 \& 2$, respectively. In the first experiment, we use an E. coli PacBio dataset and teste the accuracy using different sequencing coverages. The longest backbones generated by DBG2OLC using $10 \mathrm{x} / 30 \mathrm{x}$ data are $1.3 \mathrm{Mb}$ and $4.6 \mathrm{Mbp}$ respectively. The E. coli genome reference (4.6 $\mathrm{Mbp}$ ) can be found with accession number NC_000913. An important assembly scenario is when we have both NGS and 3GS data, we find that Sparc facilitates this hybrid assembly approach and leads to both cost efficiency and computational efficiency. After two rounds, Sparc reaches an error rate of $0.09 \%$ using only $10 \mathrm{x}$ data in a hybrid setting compared to $0.64 \%$ using PBdagcon. As expected, the quality is even better with $30 \mathrm{x}$ data $(0.02 \%)$. The error rates of using PacBio (PB) data only (i.e. non-hybrid) are higher as expected. Running the algorithms for more than two rounds lead to slightly more improvements, at the cost of more computational time. The time of running both programs for two rounds are reported in Tables $1 \& 2$.

\begin{tabular}{|c|c|c|c|c|c|c|}
\hline Program & Coverage & N50 & \# Time & Memory & Err1 & Err2 \\
\hline Sparc & 10x PB & $1.06 \mathrm{MB}$ & $110.5 \mathrm{~m}$ & $308 \mathrm{MB}$ & $1.95 \%$ & $1.51 \% 1.50 \%$ \\
\hline PBdagcon & 10x PB & $1.06 \mathrm{MB}$ & $113.0 \mathrm{~m}$ & $1.10 \mathrm{~GB}$ & $1.95 \%$ & $1.52 \% 1.51 \%$ \\
\hline Sparc & 10x Hybrid & $1.06 \mathrm{MB}$ & $110.5 \mathrm{~m}$ & $237 \mathrm{MB}$ & $0.19 \%$ & $0.09 \% 0.06 \%$ \\
\hline PBdagcon & 10x Hybrid & $1.06 \mathrm{MB}$ & $113.0 \mathrm{~m}$ & $1.23 \mathrm{~GB}$ & $1.02 \%$ & $0.58 \%$ \\
\hline Sparc & 30x PB & $4.74 \mathrm{MB}$ & $21.3 \mathrm{~m}$ & $2.30 \mathrm{~GB}$ & $0.41 \%$ & $0.16 \% 0.11 \%$ \\
\hline PBdagcon & 30x PB & $4.74 \mathrm{MB}$ & $29.3 \mathrm{~m}$ & 7.70GB & $0.49 \%$ & $0.23 \% 0.18 \%$ \\
\hline Sparc & 30x Hybrid & $4.74 \mathrm{MB}$ & $21.3 \mathrm{~m}$ & $2.14 \mathrm{~GB}$ & $0.17 \%$ & $0.02 \% 0.02 \%$ \\
\hline PBdagcon & 30x Hybrid & $4.74 \mathrm{MB}$ & $29.7 \mathrm{~m}$ & $9.58 \mathrm{~GB}$ & $0.49 \%$ & $0.18 \% 0.13 \%$ \\
\hline
\end{tabular}

Table 1. Results on an E. coli dataset using PacBio sequencing.

Sparc scales well to larger datasets; we show here the performance of Sparc and PBdagcon on a larger 20x PacBio A. thaliana dataset (genome size $120 \mathrm{Mbp}$ ). The longest backbone generated by DBG2OLC is $7.1 \mathrm{Mbp}$. Sparc finishes with $1 / 5^{\text {th }}$ time and memory compared with PBdagcon while producing more accurate results. Here a pure PacBio full genome assembly generated by MHAP (Berlin et al. 2014) is used as the reference to calculate the error rates.

Table 2. Results on an $A$. thaliana dataset using PacBio sequencing 
394

395

396

397

398

399

400

401

402

403

404

405

406

407

408

409

410

412

413

414

415

416

417

418

419

420

421

422

423

424

425

426

427

428

429

430

431

432

433

\begin{tabular}{llccccccc}
\hline Program & Coverage & N50 & $\#$ & Time & Memory & Err1 & Err2 & Err4 \\
\hline Sparc & 20x Hybrid & $2.02 \mathrm{MB}$ & 469 & $21 \mathrm{~m}$ & $1.7 \mathrm{~GB}$ & $0.36 \%$ & $0.19 \%$ & $0.17 \%$
\end{tabular}

PBdagcon 20x Hybrid $2.02 \mathrm{MB} \quad 469123 \mathrm{~m} \quad 8.9 \mathrm{~GB} \quad 0.81 \% 0.53 \% 0.47 \%$

On an Oxford Nanopore dataset, we set $k=2, g=2$ and run the consensus algorithms for four rounds in consideration of the higher error rate. The per base error rates after $1 / 2 / 4$ rounds are reported as Err1/2/4 in Table 3. The results using only Oxford Nanopore (ON) data and using hybrid 434 data are reported in rows 1,2 and rows 3,4 respectively. With Oxford Nanopore data, Sparc obtains significantly lower error rate using hybrid data. Below $0.5 \%$ error rate is reached even though the raw error rate could be as high as $40 \%$. In contrast, non-hybrid consensus generated less 437 usable results due to this excessive error rate. The longest 438 backbone in this test is $4.6 \mathrm{Mbp}$. The time of running both 439 programs for four rounds are reported in Table 3.

440

441

442

Table 3. Results on an E. coli dataset using Oxford Nanopore sequencing.

443

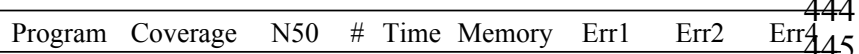

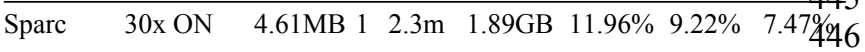
$\begin{array}{llllllllllllll}\text { PBdagcon 30x ON } \quad 4.61 \mathrm{MB} & 1 & 10.0 \mathrm{~m} & 8.38 \mathrm{~GB} & 13.70 \% & 12.96 \% & 12.864\end{array}$

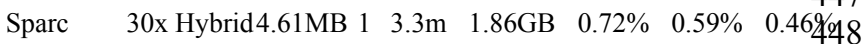
$\begin{array}{lllll}\text { PBdagcon 30x Hybrid4.61MB } 1 \quad 13.2 \mathrm{~m} \quad 9.56 \mathrm{~GB} & 11.20 \% & 10.01 \% & 9.96 \% 49\end{array}$

Sparc is relatively insensitive to different parameters and hence easy to use even for inexperienced users. We vary the parameters in the second round of the 30x PacBio E. 451 coli dataset using PacBio data only. The memory, time and 452 quality of using different $k, g$ are reported in Table 4. Using 453 a slightly larger $k$-mer size increases the per-base accuracy, 454 as more strict matches are enforced, this effect is more 455 significant on multi-ploid genomes, as different strands will have more distinct representations. However, this also increases the memory consumption because more branching nodes are created. Setting a larger $g$ helps to reduce the memory consumption. The influence of various amount of weight increase $(b=0,5,10,15)$ to NGS data in the hybrid consensus setting can be found in Table 5 . Empirically this parameter can be safely set as $b=5 \sim 15$ without compromising the accuracy. In practice we would tentatively set it to be a low values $(b=5 \sim 10)$ to more sufficiently utilize the $3 \mathrm{GS}$ data.

Table 4. Memory and quality comparisons using different $k, g$ values

\begin{tabular}{ccccc}
\hline$K$ & $g$ & Time & Memory & Error rate \\
\hline 1 & 1 & $43 \mathrm{~s}$ & $2.3 \mathrm{~GB}$ & $0.16 \%$ \\
2 & 1 & $55 \mathrm{~s}$ & $3.5 \mathrm{~GB}$ & $0.14 \%$ \\
1 & 2 & $59 \mathrm{~s}$ & $1.6 \mathrm{~GB}$ & $0.18 \%$ \\
2 & 2 & $68 \mathrm{~s}$ & $2.3 \mathrm{~GB}$ & $0.13 \%$ \\
\hline
\end{tabular}

473

Table 5. Performance of using different $b$ values

456

457

458

459

460

461

462

463

464

465

466

467

468

469

470

471

472

473

474

\begin{tabular}{lccc}
\hline Dataset & $b$ & Err1 & Err4 \\
\hline 30x PB Hybrid $E$. coli & 0 & $0.34 \%$ & $0.05 \%$ \\
30x PB Hybrid $E$. coli & 5 & $0.17 \%$ & $0.02 \%$ \\
30x PB Hybrid $E$. coli & 10 & $0.11 \%$ & $0.02 \%$ \\
30x PB Hybrid $E$. coli & 15 & $0.08 \%$ & $0.02 \%$ \\
30x ON Hybrid $E$. coli & 0 & $6.87 \%$ & $6.69 \%$ \\
30x ON Hybrid $E$. coli & 5 & $0.88 \%$ & $0.70 \%$ \\
30x ON Hybrid $E$. coli & 10 & $0.72 \%$ & $0.46 \%$ \\
30x ON Hybrid $E$. coli & 15 & $0.69 \%$ & $0.48 \%$ \\
\hline
\end{tabular}

\section{CONCLUSION}

Consensus module is a critical component in the OverlapLayout-Consensus assembly framework. With the introduction of $3 \mathrm{GS}$ technology, its importance is further raised. In this work we demonstrated a simple but efficient consensus algorithm that uses $k$-mers as building blocks and produces high quality consensus from a positionspecific $k$-mer graph. It supports hybrid sequencing data which leads to the significant improvements in both cost efficiency and computational efficiency. The proposed method is expected to significantly expand its applications in error correction and variant discovery. The consensus quality can also be enhanced further by incorporating platform specific, signal-level information.

\section{ACKNOWLEDGEMENTS}

We thank Chris Hill and Jue Ruan for help and fruitful discussions.

\section{REFERENCES}

$\mathrm{Au} \mathrm{KF}$, Underwood JG, Lee L, and Wong WH. 2012. Improving PacBio long read accuracy by short read alignment. PLoS One 7:e46679.

Berlin K, Koren S, Chin C-S, Drake J, Landolin JM, and Phillippy AM. 2014. Assembling Large Genomes with Single-Molecule Sequencing and Locality Sensitive Hashing.

Chaisson M, and Tesler G. 2012. Mapping single molecule sequencing reads using basic local alignment with successive refinement (BLASR): application and theory. BMC Bioinformatics 13:238.

Chin CS, Alexander DH, Marks P, Klammer AA, Drake J, Heiner C, Clum A, Copeland A, Huddleston J, Eichler EE, Turner SW, and Korlach J. 2013. Nonhybrid, finished microbial genome assemblies from long-read SMRT sequencing data. Nat Methods 10:563-569. 10.1038/nmeth. 2474

Edgar RC. 2004. MUSCLE: multiple sequence alignment 
with high accuracy and high throughput. Nucleic acids 528 research 32:1792-1797.

Hackl T, Hedrich R, Schultz J, and Forster F. 2014. 530 proovread: large-scale high-accuracy PacBio correction 531 through iterative short read consensus. Bioinformatics. 532 10.1093/bioinformatics/btu392

Hannenhalli S, Feldman W, Lewis HF, Skiena SS, and 534 Pevzner PA. 1996. Positional sequencing by hybridization. 535 Computer Applications in the Biosciences 12:19-24.

Huang XQ, Wang JM, Aluru S, Yang SP, and Hillier L. 537 2003. PCAP: A whole-genome assembly program. Genome 538 Research 13:2164-2170. Doi 10.1101/Gr.1390403

\section{9}

Koren S, Schatz MC, Walenz BP, Martin J, Howard JT, 540 Ganapathy G, Wang Z, Rasko DA, McCombie WR, Jarvis 541 ED, and Adam MP. 2012. Hybrid error correction and de 542 novo assembly of single-molecule sequencing reads. Nat 543 Biotechnol 30:693-700. 10.1038/nbt.2280 544 Kurtz S, Phillippy A, Delcher A, Smoot M, Shumway M, 545 Antonescu C, and Salzberg S. 2004. Versatile and open 546 software for comparing large genomes. Genome Biology 547 5:R12.

Larkin MA, Blackshields G, Brown N, Chenna R, 549 McGettigan PA, McWilliam H, Valentin F, Wallace IM, 550 Wilm A, and Lopez R. 2007. Clustal W and Clustal X version 2.0. Bioinformatics 23:2947-2948.

Laver T, Harrison J, O'Neill P, Moore K, Farbos A, Paszkiewicz K, and Studholme D. 2015. Assessing the performance of the Oxford Nanopore Technologies MinION. Biomolecular Detection and Quantification 3:1-8. Lee C, Grasso C, and Sharlow MF. 2002. Multiple sequence alignment using partial order graphs. Bioinformatics 18:452-464. DOI 10.1093/bioinformatics/18.3.452

Lee H, Gurtowski J, Yoo S, Marcus S, McCombie WR, and Schatz M. 2014. Error correction and assembly complexity of single molecule sequencing reads.

Mallat S. 2008. A wavelet tour of signal processing: the sparse way: Academic press.

Mullikin JC, and Ning ZM. 2003. The phusion assembler. Genome Research 13:81-90. Doi 10.1101/Gr.731003 Myers EW, Sutton GG, Delcher AL, Dew IM, Fasulo DP, Flanigan MJ, Kravitz SA, Mobarry CM, Reinert KH, Remington KA, Anson EL, Bolanos RA, Chou HH, Jordan CM, Halpern AL, Lonardi S, Beasley EM, Brandon RC, Chen L, Dunn PJ, Lai Z, Liang Y, Nusskern DR, Zhan M, Zhang Q, Zheng X, Rubin GM, Adams MD, and Venter JC. 2000. A whole-genome assembly of Drosophila. Science 287:2196-2204.

Myers G. 2014. Efficient Local Alignment Discovery amongst Noisy Long Reads. Algorithms in Bioinformatics: Springer, 52-67.

Nagarajan N, and Pop M. 2013. Sequence assembly demystified. Nature Reviews Genetics 14:157-167.
Pevzner PA, Tang H, and Waterman MS. 2001. An Eulerian path approach to DNA fragment assembly. Proceedings of the National Academy of Sciences 98:97489753. 10.1073/pnas. 171285098

Rausch T, Koren S, Denisov G, Weese D, Emde AK, Doring A, and Reinert K. 2009. A consistency-based consensus algorithm for de novo and reference-guided sequence assembly of short reads. Bioinformatics 25:11181124. DOI 10.1093/bioinformatics/btp 131

Ronen R, Boucher C, Chitsaz H, and Pevzner P. 2012. SEQuel: improving the accuracy of genome assemblies. Bioinformatics 28:i188-i196.

Salmela L, and Rivals E. 2014. LoRDEC: accurate and efficient long read error correction. Bioinformatics:btu538. Ye C, Hill C, Ruan J, and Ma Z. 2014. DBG2OLC: Efficient Assembly of Large Genomes Using the Compressed Overlap Graph. arXiv preprint arXiv: 14102801 .

Ye C, Ma ZS, Cannon CH, Pop M, and Yu DW. 2012. Exploiting sparseness in de novo genome assembly. BMC Bioinformatics 13 Suppl 6:S1. 10.1186/1471-2105-13-S6S1 